\title{
01.5
}

\section{Ламинарный хаос в связанных системах с запаздыванием}

\author{
(С) Д.Д. Кульминский ${ }^{1,2}$, В.И. Пономаренко ${ }^{1,3}$, М.Д. Прохоров ${ }^{1}$ \\ ${ }^{1}$ Саратовский фрилиал Института радиотехники и электроники им. В.А. Котельникова РАН, Саратов, Россия \\ ${ }^{2}$ Научно-технологический университет „Сириус“, Сочи, Россия \\ ${ }^{3}$ Саратовский национальный исследовательский государственный университет им. Н.Г. Чернышевского, Саратов, Россия \\ E-mail: mdprokhorov@yandex.ru
}

Поступило в Редакцию 1 октября 2021 г.

В окончательной редакции 3 ноября 2021 г.

Принято к публикации 4 ноября 2021 г.

\begin{abstract}
Исследована возможность существования ламинарного хаоса в связанных системах с запаздывающей обратной связью. Рассмотрены случаи однонаправленной и взаимной связи систем с запаздыванием. Впервые показано, что ламинарный хаос может существовать не только в системе с переменным временем задержки, но и в системе с постоянным временем задержки, если она связана с системой, находящейся в режиме ламинарного хаоса.
\end{abstract}

Ключевые слова: системы с запаздыванием, ламинарный хаос, связанные осцилляторы, синхронизация.

DOI: 10.21883/PJTF.2022.04.52077.19044

Наличие запаздывающей обратной связи типично для многих систем различной природы [1,2], что обусловливает большой интерес к исследованию систем с запаздыванием в самых разных областях науки $[3,4]$. При этом в большинстве работ по изучению систем с запаздыванием рассматривается случай постоянного времени задержки. Однако исследование систем с переменным временем задержки представляет не меньший интерес, поскольку на практике задержка может флуктуировать в силу самых разнообразных причин [5,6], что существенно усложняет динамику системы [7,8]. Модуляция времени задержки оказывается полезной для ряда прикладных задач. Например, использование хаотических генераторов с переменным временем задержки в системах передачи информации позволяет повысить их конфиденциальность $[9,10]$ по сравнению с системами связи на основе хаотических генераторов с запаздывающей обратной связью, имеющих постоянное время задержки $[11,12]$.

В системах с переменным временем задержки недавно был обнаружен новый тип хаотического поведения, который был назван ламинарным хаосом [13]. Такой тип поведения характеризуется чередованием различных ламинарных фаз, в течение которых динамическая переменная остается практически постоянной, а при переходе из одной ламинарной фазы в другую ее значение меняется хаотически. Существование ламинарного хаоса было продемонстрировано не только на численных примерах, но и в экспериментальных системах: оптоэлектронном генераторе с изменяющимся во времени запаздыванием [14], электронном генераторе с переменной задержкой [15] и радиотехническом генераторе с запаздывающей обратной связью, время задержки которого модулируется внешним гармоническим сигналом [16].

До сих пор явление ламинарного хаоса наблюдалось только в одиночных автоколебательных системах с запаздыванием при определенных условиях изменения времени задержки [13-17]. В настоящей работе мы впервые показали, что ламинарный хаос может существовать не только в системе с переменным временем задержки, но и в системе с постоянным временем задержки, если она связана с системой, находящейся в режиме ламинарного хаоса.

Рассмотрим систему с запаздыванием, которую в отсутствие связи с другими системами можно представить в виде кольца, состоящего из трех элементов (нелинейного, инерционного и задержки), и описать следующим дифференциальным уравнением первого порядка с запаздыванием:

$$
\varepsilon \dot{x}(t)=-x(t)+f(x(t-\tau)),
$$

где $\varepsilon$ - параметр, характеризующий инерционные свойства системы, $\tau$ - время задержки, $f-$ нелинейная функция. В системе (1) время задержки является постоянным. При соответствующем выборе нелинейной функции (например, квадратичной или синусоидальной) система (1) демонстрирует при $\tau \gg \varepsilon$ хаотические колебания динамической переменной $x(t)$ [1]. Поскольку во временно́й реализации $x(t)$ при этом отсутствуют ламинарные участки, на которых $x(t)$ остается постоянной, такие хаотические колебания были названы в [13] турбулентным хаосом.

При периодически изменяющемся времени задержки

$$
\tau(t)=\tau_{0}+\tau_{m} \sin (2 \pi \nu t),
$$

где $\tau_{0}-$ среднее значение времени задержки, $\tau_{m}-$ глубина модуляции времени задержки, а $v$ - частота модуляции, система будет описываться уравнением

$$
\varepsilon \dot{x}(t)=-x(t)+f(x(t-\tau(t)))
$$

и может демонстрировать качественно другой тип хаотической динамики, названный ламинарным хаосом $[13,14]$, 

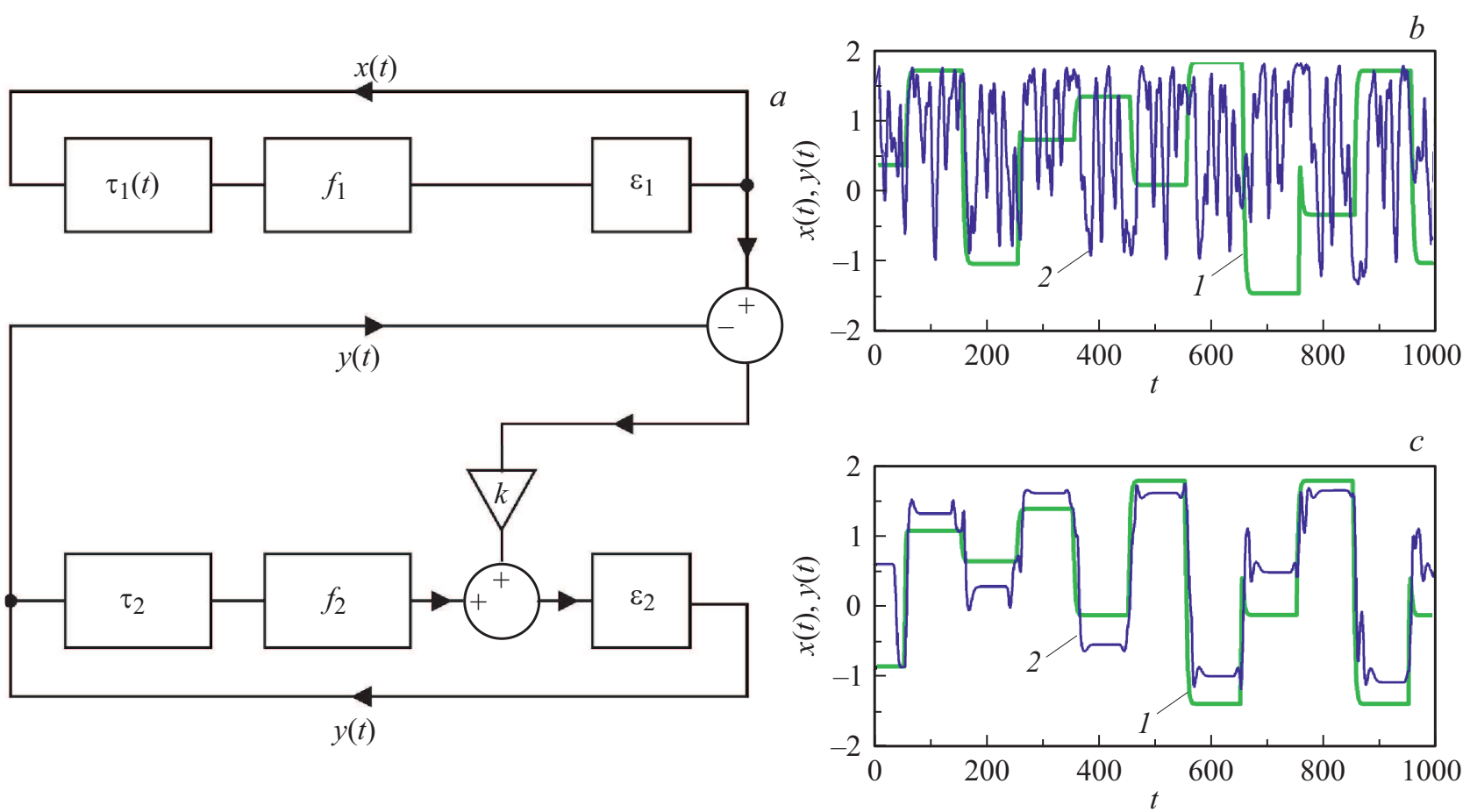

Рис. 1. $a-$ блок-схема двух однонаправленно связанных систем с запаздыванием. Элементы, обозначенные $\tau_{1}(t)$ и $\tau_{2}, f_{1}$ и $f_{2}$, $\varepsilon_{1}$ и $\varepsilon_{2}$, обеспечивают соответственно задержку, нелинейное и инерционное преобразования колебаний, а элемент $k$ определяет величину однонаправленной связи. $b$ и $c$ - временны́е реализации колебаний $x(t)(1)$ и $y(t)(2)$ при $k=0$ и 0.55 соответственно.

при котором реализация $x(t)$ имеет горизонтальные плато.

Рассмотрим теперь две системы с запаздыванием, из которых одна имеет постоянное время задержки и находится в режиме турбулентного хаоса, а другая имеет переменное время задержки и находится в режиме ламинарного хаоса, и свяжем эти системы между собой. Системы с запаздыванием могут быть связаны между собой различными способами, которые отличаются не только типом связи (линейная, диффузионная, запаздывающая), но и тем, в какую точку кольцевой системы с запаздыванием подается сигнал от другой системы [18]. Так, в систему, описываемую уравнением (1), сигнал связи можно подать между инерционным элементом (фильтром) и линией задержки, между линией задержки и нелинейным элементом, между нелинейным элементом и фильтром. Каждый из этих случаев будет описываться своим уравнением [18].

На рис. 1, a приведена блок-схема двух однонаправленно связанных систем с запаздыванием (1) и (3) для случая, когда сигнал диффузионной связи подается на ведомую систему между нелинейным элементом и фильтром. При таком способе связи ведущая система описывается уравнением $\varepsilon_{1} \dot{x}(t)=-x(t)+f_{1}\left(x\left(t-\tau_{1}(t)\right)\right)$, а ведомая система - уравнением

$$
\varepsilon_{2} \dot{y}(t)=-y(t)+f_{2}\left(y\left(t-\tau_{2}\right)\right)+k(x(t)-y(t)),
$$

где $k$ - коэффициент связи.
Параметры ведущей и ведомой систем были выбраны следующими: $\tau_{1}(t)=1+0.2 \sin (2 \pi t)$, $\tau_{2}=1, \quad \varepsilon_{1}=\varepsilon_{2}=0.03, \quad f_{1}=\lambda_{1}-x^{2}, \quad f_{2}=\lambda_{2}-y^{2}$, где $\lambda_{1}=\lambda_{2}=1.82$ - параметры нелинейности, шаг интегрирования $\Delta t=0.01$. На рис. $1, b$ приведены временны́е реализации колебаний $x(t)$, соответствующие режиму ламинарного хаоса, и колебаний $y(t)$ при $k=0$, соответствующие режиму турбулентного хаоса. С увеличением силы связи режим турбулентного хаоса в ведомой системе начинает разрушаться. Во временно́й реализации $y(t)$ появляются участки ламинарного хаоса, длительность которых растет с ростом $k$. На рис. 1, $c$ приведены временны́е реализации колебаний $x(t)$ и $y(t)$ при $k=0.55$. Видно, что горизонтальные участки в реализации $y(t)$ короче, чем в реализации $x(t)$. Кроме того, в ведомой системе при $k=0.55$ участки ламинарного хаоса во временно́й реализации $y(t)$ перемежаются с участками турбулентного хаоса, которые не показаны на рис. 1,c. Коэффициент корреляции $R$ между ведущей и ведомой системами равен 0.82 при $k=0.55$. При дальнейшем увеличении силы связи колебания $x(t)$ и $y(t)$ становятся все более схожими между собой, и при $k>1$ они становятся практически идентичными, т.е. происходит полная синхронизация ведущей и ведомой систем. Коэффициент корреляции $R$ при этом близок к единице.

Рассмотрим более сложный случай взаимной связи систем с запаздыванием. На рис. 2, $a$ приведена блоксхема двух взаимно связанных систем с запаздыванием 

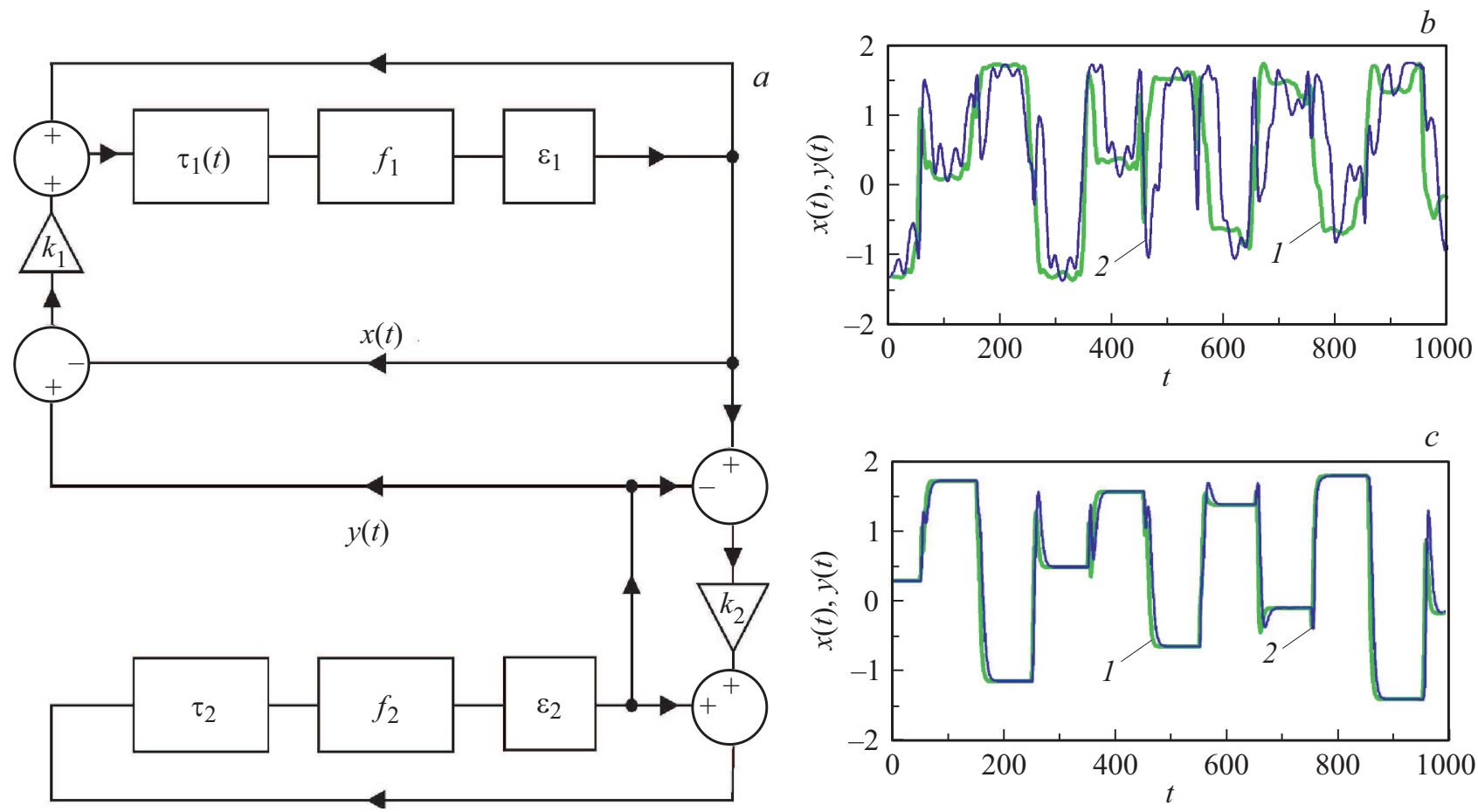

Рис. 2. $a-$ блок-схема двух взаимно связанных систем с запаздыванием. Элементы, обозначенные $\tau_{1}(t)$ и $\tau_{2}, f_{1}$ и $f_{2}, \varepsilon_{1}$ и $\varepsilon_{2}$, обеспечивают соответственно задержку, нелинейное и инерционное преобразования колебаний, а элементы $k_{1}$ и $k_{2}$ определяют величину связи между системами. $b$ и $c-$ временны́е реализации колебаний $x(t)(1)$ и $y(t)(2)$ при $k_{1}=k_{2}=0.1$ и 0.8 соответственно.

(1) и (3) для случая, когда сигнал диффузионной связи подается на обе системы между фильтром и линией задержки. При таком способе связи системы описываются уравнениями

$$
\begin{aligned}
\varepsilon_{1} \dot{x}(t) & =-x(t)+f_{1}\left(x\left(t-\tau_{1}(t)\right)\right. \\
+ & \left.k_{1}\left[y\left(t-\tau_{1}(t)\right)-x\left(t-\tau_{1}(t)\right)\right]\right), \\
\varepsilon_{2} \dot{y}(t) & =-y(t)+f_{2}\left(y\left(t-\tau_{2}\right)\right. \\
& \left.+k_{2}\left[x\left(t-\tau_{2}\right)-y\left(t-\tau_{2}\right)\right]\right),
\end{aligned}
$$

где $k_{1}$ и $k_{2}$ - коэффициенты связи. Параметры обеих систем выбраны такими же, как в рассмотренном выше случае однонаправленной связи, поэтому при $k_{1}=k_{2}=0$, временны́е реализации колебаний $x(t)$ и $y(t)$ имеют такой же вид, как на рис. $1, b$, т.е. первая система находится в режиме ламинарного хаоса, а вторая - в режиме турбулентного хаоса. На рис. $2, b$ приведены временнь́е реализации колебаний $x(t)$ и $y(t)$ при $k_{1}=k_{2}=0.1$. При такой силе связи наблюдается заметное изменение колебательных режимов в обеих системах по сравнению со случаем отсутствия связи. Случай сильной связи $\left(k_{1}=k_{2}=0.8\right)$ представлен на рис. 2, c, на котором обе системы демонстрируют ламинарный хаос и синхронизованы между собой.

Итак, нами впервые показано, что ламинарный хаос может существовать в системе с постоянным временем задержки, если она связана с системой, находящейся в режиме ламинарного хаоса. Таким образом, ламинарный хаос можно получить не только с помощью модуляции времени задержки, но и за счет введения связи между системами с запаздыванием.

\section{Финансирование работы}

Работа выполнена при поддержке Российского фонда фундаментальных исследований (проект № 19-0200071).

\section{Конфликт интересов}

Авторы заявляют, что у них нет конфликта интересов.

\section{Список литературы}

[1] В.И. Пономаренко, М.Д. Прохоров, А.С. Караваев, Б.П. Безручко, Системы с запаздыванием (реконструкция моделей и их приложение) (Изд-во Саратов. ун-та, Саратов, 2016).

[2] G.A. Bocharov, F.A. Rihan, J. Comput. Appl. Math., 125, 183 (2000). DOI: 10.1016/S0377-0427(00)00468-4

[3] Y. Kuang, Delay differential equations with applications in population dynamics (Academic Press, Boston, 1993).

[4] T. Erneux, Applied delay differential equations (SpringerVerlag, N.Y., 2009). 
[5] J. Martínez-Llinás, X. Porte, M.C. Soriano, P. Colet, I. Fischer, Nature Commun., 6, 7425 (2015). DOI: 0.1038/ncomms 8425

[6] D. Müller, A. Otto, G. Radons, Phys. Rev. E, 95, 062214 (2017). DOI: 10.1103/PhysRevE.95.062214

[7] D.V. Senthilkumar, M. Lakshmanan, Chaos, 17, 013112 (2007). DOI: $10.1063 / 1.2437651$

[8] L. Lazarus, M. Davidow, R. Rand, Int. J. Nonlinear Mech., 78, 66 (2016). DOI: 10.1016/j.ijnonlinmec.2015.10.005

[9] D. Ghosh, S. Banerjee, A.R. Chowdhury, Europhys. Lett., 80, 30006 (2007). DOI: 10.1209/0295-5075/80/30006

[10] A.S. Karavaev, D.D. Kulminskiy, V.I. Ponomarenko, M.D. Prokhorov, Int. J. Bifurc. Chaos Appl. Sci. Eng., 25, 1550134 (2015). DOI: 10.1142/S0218127415501345

[11] V.I. Ponomarenko, M.D. Prokhorov, Phys. Rev. E, 66, 026215 (2002). DOI: 10.1103/PhysRevE.66.026215

[12] V.I. Ponomarenko, M.D. Prokhorov, A.S. Karavaev, D.D. Kulminskiy, Nonlinear Dyn., 74, 1013 (2013). DOI: $10.1007 / \mathrm{s} 11071-013-1019-0$

[13] D. Müller, A. Otto, G. Radons, Phys. Rev. Lett., 120, 084102 (2018). DOI: 10.1103/PhysRevLett.120.084102

[14] J.D. Hart, R. Roy, D. Müller-Bender, A. Otto, G. Radons, Phys. Rev. Lett., 123, 154101 (2019). DOI: 10.1103/PhysRevLett.123.154101

[15] T. Jüngling, T. Stemler, M. Small, Phys. Rev. E, 101, 012215 (2020). DOI: 10.1103/PhysRevE.101.012215

[16] Д.Д. Кульминский, В.И. Пономаренко, М.Д. Прохоров, Письма в ЖТФ, 46 (9), 16 (2020). DOI: 10.21883/PJTF.2020.09.49366.18218 [D.D. Kul'minskii, V.I. Ponomarenko, M.D. Prokhorov, Tech. Phys. Lett., 46 (5), 423 (2020). DOI: 10.1134/S1063785020050090].

[17] D. Müller-Bender, A. Otto, G. Radons, J.D. Hart, R. Roy, Phys. Rev. E, 101, 032213 (2020). DOI: 10.1103/PhysRevE.101.032213

[18 ] M.D. Prokhorov, V.I. Ponomarenko, Phys. Rev. E, 72, 016210 (2005). DOI: 10.1103/PhysRevE.72.016210 